\title{
Neonatal brainstem dysfunction
}

INSERM

\section{Source}

INSERM. (1999). Orphanet: an online rare disease and orphan drug data base. Neonatal brainstem dysfunction. ORPHA:137929

Neonatal brainstem dysfunction is a rare neurologic disease characterized by the association of suction-swallowing dysfunction, abnormal laryngeal sensitivity and motility (manifesting with dyspnea or obstructive apnea-hypopnea), gastroesophageal reflux (generally resistant to medication) and cardiac vagal overactivity (e.g. brachycardia, vasovagal episodes) of varying degrees of severity. Impaired social interaction has also been reported. 